\title{
Dynamical Spot Queries to Improve Specificity in P450s based Multi-Drugs Monitoring
}

\author{
Sandro Carrara*, Andrea Cavallini, Abhishek Garg, Giovanni De Micheli \\ Swiss Federal Institute of Technology - Lausanne (EPFL) CH-1015 Lausanne (CH) - * sandro.carrara@epfl.ch
}

\begin{abstract}
Personalized therapy requires accurate and frequent monitoring of drugs metabolic response in living organisms during drug treatments. In case of high risk side effects, e.g. therapies with interfering anti-cancer molecules cocktails, direct monitoring of the patient's drug metabolism is essential as the metabolic pathways efficacy is highly variable on a patient-bypatient basis. Moreover, anti-cancer pharmacological treatments are often based on cocktails of different drugs. Currently, there are no fully mature biochip systems to monitor multi-panel drugs amount in blood or in serum. The aim of this paper is to investigate the complexity of multiple drugs detection for pointof-care systems to be used in personalized therapy. Probes molecules for the biochip are the $\mathbf{P 4 5 0}$ enzymes as they have key role in drugs metabolism. Multiple drugs detection is carried out both by simulations and electrochemical experiments. Drugs specificity enhancement is investigated considering components decomposition of peak as registered in cyclic voltammetry acquisitions. This investigation has the aim to identify crucial aspect in VLSI design of fully-electronics biochip development in this field.
\end{abstract}

\section{INTRODUCTION}

New frontiers in personalized therapy also envisage the development of new tools for fast, easy-to-use, low cost, and point-of-care analysis of drugs efficacy on the patients. The demand for new tools arose because still most effective drug therapies for major diseases provide benefit only to a fraction of patients, typically in the 20 to $50 \%$ range [1]. One of the reasons relies on the patient's genetic polymorphism. For example, polymorphism in cytochrome P450 2D6 phenotype [2] causes overdosing in "poor metabolizers" and underdosing in "ultrafast metabolizers", and explains why approximately $7 \%$ of hospitalized patients have serious adverse drug reactions [1]. Some of the commonly used drugs for hypertensions or anti-cancer treatments (e.g. metoprolol and tamoxifen) are metabolized by this cytochrome. Therefore, it is being increasingly recognized that any drug therapy needs to be personalized to the individual patient [3, 4]. The only available monitoring system in the present stateof-the-art for personalized therapy is a check of the genetic predisposition of patients. In order to know the risk for subtherapeutic or toxic drug concentrations, a genetic test is done on alleles, which correspond to patient genetic predisposition for expressing the $\mathrm{P} 450$ proteins. For example, mutations causing nonfunctional alleles may lead to a complete deletion of the CYP2D6 gene. For this aim, a genetic test based on microarray has been introduced into the marked by Roche: the AmpliChip CYP450 [5]. It is the first FDA-cleared test for analysis of only CYP2D6 and CYP2C19, two genes in the cytochrome $\mathrm{P} 450$ system that can greatly influence drug metabolism. By testing patient's genotyping using AmpliChip, the patient can be classified as poor, intermediate, extensive, or ultra-rapid metabolizer. This classification affects the actual amount of mean plasma concentration after a single drug dose, as demonstrated in the case of nortriptyline [6]. However, this chip can only "predict" the patient's phenotype while human metabolism may vary on a daily basis. Thus, in order to individually optimize an ongoing drug therapy, it is required to know drug concentrations in the patient's body at the moment of the pharmacological cure. It is a major weakness in the concept of personalized medicine that the current available tools cannot acquire this information on real-time basis. Therapeutic drug monitoring, i.e. quantification of drug concentrations after administration to see whether the desired concentration range has been reached, is available only in large clinical chemistry labs. These chemistry labs typically offer this service for 20 drugs, and specialized labs at universities may run routine methods for 50 drugs, but they provide the measurements with timing of days.

A reliable point-of-care technology to monitor multiple drug compounds in patients for personalized treatments is still not present either into the market or into the scientific state-ofthe-arte. In this paper, we propose a new method for designing biochips to measure multiple drugs concentration in real-time. The proposed system is based on multi-panel cytochromes P450 and opens the possibility to contemporary multi-drugs detection directly in patients' serum. Biosensors based on individual $\mathrm{P} 450$ proteins have already been proposed to develop novel drug screening tools [7, 8, 9]. Electrodes nanostructuring has been shown to enhance P450 based sensitivity by using zirconium dioxide [10] as well as gold [11] nanoparticles, and carbon nanotubes [12]. However, the development of P450 based chip for multi- drugs screening meets serious drawbacks as each P450 cytochrome detects many drugs and different P450s may detect the same drug. Aim of the present research is to identify these drawbacks and propose possible biochip design to overcome them.

\section{P450 BASED ARRAY SYSTEM}

A P450 based biochip array provides a very powerful tool for personalization of drug therapy because of the key role of this proteins family in the human metabolism [13]. This section describes the P450 cytochromes, some of the drug substrates, and P450-drug interactions from the point of view of electrochemical investigation.

\section{A. P450 Protein family}

Cytochrome P450 (also abbreviated with CYP, or CYP450) is a diverse super-family of heme-proteins found in Bacteria, Archaea and Eukaryotes [14]. Cytochromes P450 are involved in metabolism of a plethora of both exogenous and endogenous compounds. 


\begin{tabular}{|c|c|c|}
\hline P450-2B4 & P450-3A4 & P450-2C9 \\
\hline Benzphetamine (BZ) - anti-obesity & Cyclophosphamide (CP) - anti-cancer agent & Torsemide (TM) - Diuretic \\
\hline 7-pentoxyresorufin (PX) - anti-obesity & Dextromethorphan (DX) - analgesic & Diclofenac (DC) - anti-inflammations \\
\hline Aminopyrine (AP) - anti-inflammatory/analgesic & Midazolam (MZ) - Anticonvulsant/sedative & S-Warfarin (SW) - anti-coagulant \\
\hline
\end{tabular}

Table 1: interaction map of the biochip probe molecules and the target molecules considered in this study. The probes are different proteins of the cytochromes P450 family while the targets are commonly used drugs for cardiovascular, antidepressant, or anti-cancer therapies. $\mathrm{N}$ means no interaction.

Usually, they form part of multi-component electron transfer chains, called P450-containing systems. Most P450 can metabolize multiple substrates, and many can catalyze multiple reactions, which accounts for their central importance in metabolizing the potentially endless variety of endogenous and exogenous molecules.

In drug metabolism, cytochrome $\mathrm{P} 450$ is probably the most important element of oxidative metabolism (also known as Phase I metabolism) in humans (metabolism in this context being the chemical modification or degradation of chemicals including drugs and endogenous compounds). Up to 57 genes and more than 59 pseudo-genes of the human genome encode 18 families of cytochrome P450 and 43 subfamilies. Heme proteins cytochromes $\mathrm{P} 450$ are capable of metabolizing a vast number and variety of organic substrates, estimated to be in the region of 200,000 chemicals or more, and involving around 60 distinct classes of biotransformation reactions. Metabolism mediated by cytochrome P450 enzymes plays a key role in the development of new drugs and in individual drug therapy.

P450 proteins are known to metabolize all the drugs. For example, the protein CYP2D6, formerly named debrisoquine hydroxylase, is involved in the oxidative metabolism of about $25 \%$ of commonly prescribed drugs [15]. A high range of inter-individual and inter-ethnic variability characterizes the enzyme activity, mainly caused by genetic polymorphisms. These polymorphisms can result in 30- to 40 -fold differences in substrate drugs clearance, resulting in concentrations outside the therapeutic range in some treated patients. As a consequence, such differences in CYP2D6 activity may lead not only to severe adverse effects in clinical therapy, e.g., in antidepressant therapy [16], but also to non-response to medications, such as no observable analgesic effect after low doses of codeine in poor metabolizers (PM) [17]. Starting from the first description of a polymorphism in debrisoquine hydroxylation [18] until today, more than 80 allelic variants have been described [19]. The CYP2D6 genotype has a major impact on enzymatic activity. Today genotyping may be performed by arrays such as the AmpliChip CYP450 Test [5], which identifies 20 CYP2D6 alleles and seven CYP2D6 duplications. However, genotyping alone may not exactly predict a person's phenotype. Moreover, the CYP2D6 activity is modified by certain environmental factors, e.g., infections and nutritional habits [20]. Therefore, to obtain a precise picture of a person's actual enzymatic activity, adequate probe drugs have to be administered followed by consecutive measurements of their concentrations and those of CYP2D6dependent metabolites in body fluids [21].

\section{B. Drugs substrates}

As a prelude to any individualized drug therapy aimed at preventing lack of response to medication or adverse effects due to different levels of P450 activity, genotyping as well as phenotyping have to be considered. The integrated multi-panel bio-sensor array platform can play a strategic role in monitoring phenotypic drug metabolism. Different P450 cytochromes have to be considered to develop a multi-panel detection of drugs content in human serum. The same P450 isoform may detect different drug compounds while the same drug compound is detected by different isoforms. To investigate the cross correlation between P450 enzymes to be considered as probe molecules for point-of-care biochips and drugs for screening in personalized therapies, we will consider here three different cytochromes and their drugs substrates. The enzyme P450 in the isoform 2B4 detects benzphetamine (BZ), aminopyrine (AP) and 7-pentoxyresorufin (PX). The benzphetamine is an anoretic compound closely related to amphetamine. It is often used in anti-obesity therapy. The aminopyrine is a drug with analgesic, anti-inflammatory, and antipyretic properties. The 7-pentoxyresorufin is indicated for infections, hischemia, hypertrophy, obesity, etc. The isoform P450 3A4 detects cyclophosphamide (CP), dextromethorphan (DX) and midazolam (MZ). Cyclophosphamide is an anticancer agent; dextromethorphan is an analgesic, while midazolam is a benzodiazepine derivative. It has powerful anxiolytic, amnestic, hypnotic, anticonvulsant, skeletal muscle relaxant and sedative properties. Finally, the isoform 2C9 detects torsemide (TM), diclofenac (DC), and S-Warfarin (SW). They are used for cardiovascular, anti-inflammations, anti-coagulant therapies, respectively. S-Surfamide is also used as anti-arrhythmic in cardiovascular treatments. Table 1 summarizes the P450/drugs interactions used in the present study. The indicated P450 enzymes may be used to functionalize different electrodes spots in order to develop a electrochemical Biochip for multiple drugs monitoring.

\section{CV Signature}

An electrical signature is required to investigate the $\mathrm{P} 450$ drugs interactions and to consider them for point-of-care biochip in drugs screening and monitoring. In particular, when more than one drug compound interacts with a cytochromes P450 onto the biochip electrodes, the biochip have to register a clear electrical signatures. This signature enables us to identify the drugs and to estimate its amount in the presence of other compounds cross interacting with the same biochip spot. Figure 1 show a typical electrochemical current registered in case of multiple electrolytes voltammetry. 


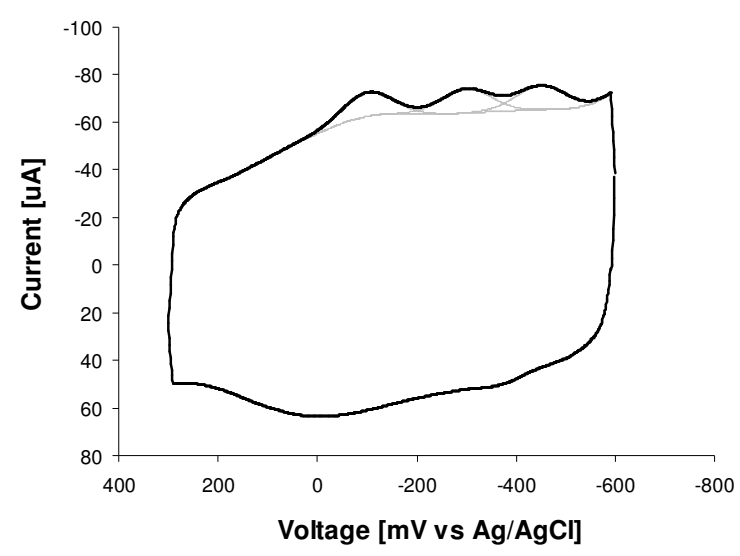

Fig. 1. Electrochemical signature of three well separated electrolyte components as registered in a Cyclic Voltammograms.

As clearly shown by the picture, the current shape upon the scanned voltage may be analyzed by considering Gaussian components. In fact, the cyclic voltammogram show a current peak that corresponds to the electrochemical signal registered during the redox reaction catalyzed by the cytochrome onto the single drug. If more drugs are present into the sample, then the voltammogram presents more than one peak. Each peak accounts for the different drug contribution to the electrolyte. This multiple voltammogram' features is what we will refer to as the "electrochemical signature" of the drug contributions. Any multi-panel biochip needs to have implemented cyclic voltammograms signature features extraction in order to improve the specificity of single drug detection.

\section{MATERIALS AND METHODS}

\section{A. The Simulator}

It is well known that multiple electrolytes produce multiple peaks in the single Cyclic Voltammograms [22]. A similar situation is modeled by the following equation:

$$
i(V)=i_{C}(V)+\sum_{\forall k} A_{k} e^{-\frac{\left(V-V_{k}\right)^{2}}{\sigma_{k}{ }^{2}}},
$$

where the first term accounts for the charging current, and the following sum of Gaussian curves accounts for all the Faradic currents generated by P450 multiple interactions with different drugs. Faradic currents from detection of different enzyme' substrates are simulated by considering the P450 peaks shift (shift in $V_{k}$ ) occurring in cyclic voltammograms when drugs are detected, while the charging current $\left(i_{c}(V)\right)$ were acquired in real experiments with physiological buffer (PBS) as electrolyte but in the absence of any cytochrome onto the working electrodes. This current was assumed as base-line for simulated voltammograms. Peaks corresponding to electrochemical drugs detection as registered in cyclic voltammetry are generated by Gaussian curves. The position of each individual Gaussian are selected by information obtained from literature or registered in voltammetric experiments. Peak areas (parameters $A_{k}$, and $\sigma_{k}$ ) are instead estimated considering typical peak features in real experiments and ratio of kinetic parameters of the simulated drugs.

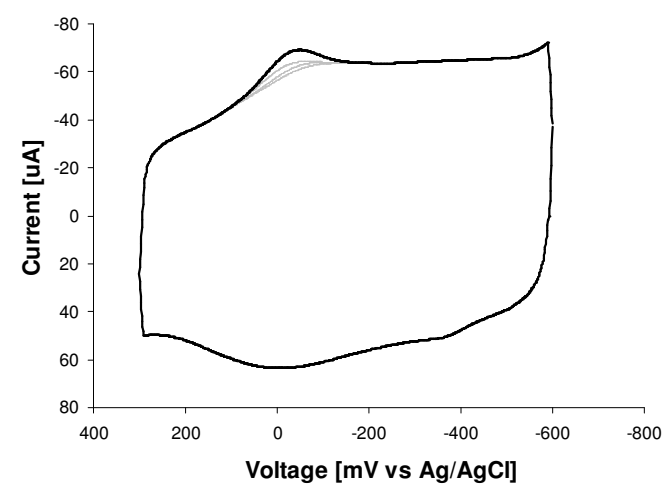

Fig. 2. Simulated voltammogram of a P450 2C9 sensor during the contemporary detection of TM, DC, SW, three not well resolved drugs.

\section{B. The Sensors}

The sensors were fabricated by using commercially available screen-printed electrodes and by using different P450 proteins and, in some cases, carbon nanotubes, too. Carbon paste screen-printed electrodes were purchased from Dropsens (model DRP-110). The electrodes were made of a graphite working electrode (area, $13 \mathrm{~mm}^{2}$ ), a graphite counter electrode and an $\mathrm{Ag} / \mathrm{AgCl}$ reference electrode. The total area of the cell is $22 \mathrm{~mm}^{2}$. Cytochrome P450 3A4 microsomes were purchased from Sigma-Aldrigh and used without modifications. CP and DX drug (from Sigma) were diluted in PBS $100 \mathrm{mM}$ pH 7.4 in aliquots of $50 \mathrm{nM}, 5 \mu \mathrm{M}, 500 \mu \mathrm{M}, 50$ $\mathrm{mM}$. Experiments were also carried out by using the cytochromes P450 2B4. In this case, the cytochrome was isolated and purified from the microsomal fraction of rabbit [27]. Cytochrome and benzphetamine (BZ) substrates were received from Russia. For both cytochromes, sensing electrodes were prepared by spreading $\mathrm{P} 450$ solution onto working electrode and incubating at $37^{\circ} \mathrm{C}$ overnight. In same cases, the effects of electrodes nano-structuring were also tested. Multi walled carbon nanotubes (MWCNT), diameter $10 \mathrm{~nm}$, length $1-2 \mu \mathrm{m}, \mathrm{COOH}$ content $5 \%$ ) were purchased in powder (95\% purity) from DropSense (Spain), diluted in chloroform to the concentration of $1 \mathrm{mg} / \mathrm{ml}$ [7] and then sonicated for 20 minutes in order to break macro-aggregates. In case of nano-structuring, the working electrodes were prepared in a 3 steps procedure: $60 \mu \mathrm{l}$ of CNT solution were drop casted onto the working electrode and dried. Electrodes were then incubated in milliQ water overnight in order to hydrate the nanostructure. The day After the P450 solution were spread onto working electrode and incubated at $37^{\circ} \mathrm{C}$ overnight.

In all the cases, the electrochemical response was investigated by cyclic voltammetry under aerobic conditions. Voltammograms were acquired by using a Versastat 3 potentiostat (Princeton Applied Technologies). The electrode was covered with $100 \mu \mathrm{l}$ of PBS $100 \mathrm{mM}$ at pH 7.4 or with the same volumes of PBS added to $1 \mu \mathrm{l}$ of drugs samples. The cyclic voltammograms were acquired with potential sweeps between -600 and $+300 \mathrm{mV}$ vs $\mathrm{Ag} / \mathrm{AgCl}$ by using different scan rates ranging from 20 to $100 \mathrm{mV} / \mathrm{sec}$. 


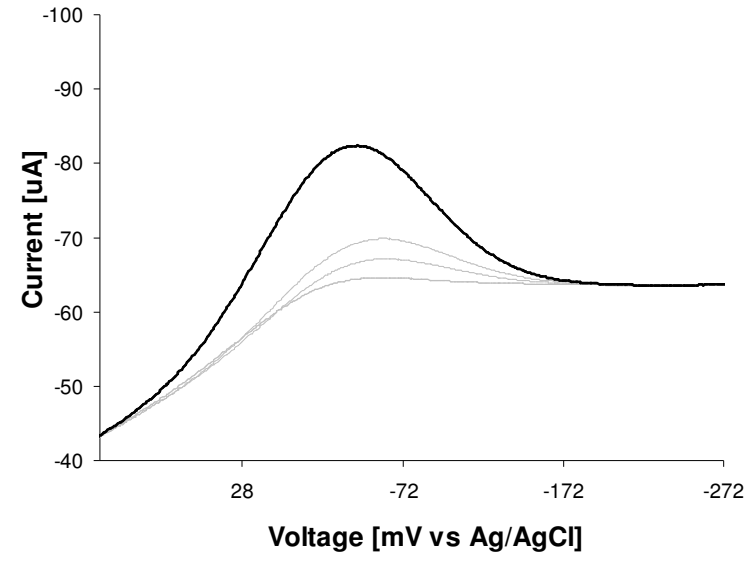

Fig. 3. Zoom in the region of interest of the Simulated Voltammogram of P450 $2 \mathrm{C} 9$ sensor during the contemporary detection of TM, DC, SW.

\section{Simulation Results}

\section{A. P450 2C9-Multiple Drugs Detection}

Detection of different drug substrates are simulated by considering the P450 peak shift occurring in cyclic voltammograms when drugs are detected. In case of the cytochrome $2 \mathrm{C}$, peak positions of the three considered drugs are slightly different. They are $-19,-41,-36 \mathrm{mV}$ for TM, DC, and SW, respectively, in presence of oxygen [23]. Figure 3 shows the cyclic voltammogram outlined by simulations. The three individual drugs contributions into voltammogram of Figure 2 are not as well resolved as those in Figure 1. This is due to the close proximity of the redox peaks of the three compounds when the redox is catalyzed by the cytochrome. Nevertheless, Figure 3 shows that the individual substrate contributions are clearly present under the voltammogram peak. These drugs followed similar Michaelis-Menten kinetics. In fact, the $\mathrm{Km}$ are $11.4 \pm 0.1$ and $6.8 \pm 0.01 \mathrm{mM}$ for $\mathrm{TM}$ and $\mathrm{DC}$, and the $\mathrm{V}_{\max }$ are $11.5 \pm 0.5$ and $23.2 \pm 0.01$ $\mathrm{pmol} / \mathrm{min} /$ for pmol of P450 [23], while the Km of SW is close to $6 \mathrm{mM}$ [24]. Thus, for drugs concentration in similar range of the different drugs, the peak registered while acquiring a cyclic voltammogram on the biochip spot will present contribution from the three compounds. For example, in the simulation of Figure 3, the drug concentrations are 20, 20, and $15 \mathrm{mM}$ for TM, DC, SW, respectively.

\section{EXPERIMENTAL RESULTS}

\section{A. $\quad \mathrm{P} 4503 A 4-$ Multiple Drugs Detection}

To validate the simulations, experiments were carried out with screen-printed carbon paste electrodes. Figure 4 shows the zoon in the region of interest of a cyclic voltammogram, acquired with a scan rate of $20 \mathrm{mV} / \mathrm{sec}$, during contemporaneous detection of Cyclophosphamide (CP), and Dextromethorphan (DX). The signal was analyzed by considering the individual peak positions registered in similar experiments where only an individual drug was present at a time. The peak positions are $-414.4 \pm 0.1 \mathrm{mV}$ and $-421.5 \pm 0.1$ $\mathrm{mV}$ for $\mathrm{CP}$ and $\mathrm{DX}$. The drug concentrations during the experiment were equal to $400 \mathrm{mM}$ for both the $\mathrm{DX}$ and $\mathrm{CP}$.

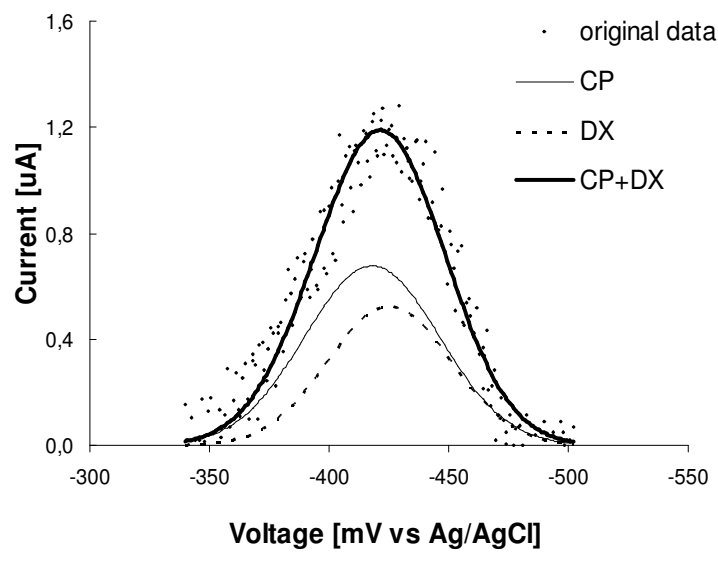

Fig. 4. Zoom in the region of interest of the a Voltammogram acquired with P450 3A4 sensor during the contemporary detection of CP and DX drugs.

The two drug compounds present $\mathrm{Km}$ in the same magnitude order. The $\mathrm{Km}$ and $\mathrm{Vmax}$ are $595 \mathrm{uM}$ and $26.9 \mathrm{pmol} / \mathrm{min} /$ for pmol of P450 for CP [25], while they are $268 \mathrm{uM} 1.74$ $\mathrm{pmol} / \mathrm{min} /$ for $\mathrm{pmol}$ of $\mathrm{P} 450$ for DX [26]. In fact, detection peaks registered in the voltammograms are well explained by fitting accounting for both the $\mathrm{DX}$ and $\mathrm{CP}$ contributions with a small chi square. In case of Figure 4, it is equal to 2.32 .

\section{B. P450 2B4 - Single Drug Detection}

Results of these experiments are reported in Figures 5, and 6. Figure 5 summarize the BZ detection as registered in cyclic voltammograms while the Figure 6 presents the effect of electrodes nano-structuring in chronoamperometric experiments. The chronoamperometries were registered by applying a constant potential equal to $-400 \mathrm{mV}$ and registering the current upon the time in sample were an increasing amount of BZ was added in different steps. Figure 5 clearly shows as the single component peak is growing up upon BZ concentration. Figure 6 shows the calibration curve acquired in cronoamperometry. These curves demonstrate that the drug detection is enhanced by using nano-structured biochip electrodes. The gain is close to one order of magnitudes, as already registered by using $\mathrm{P} 450$ proteins and carbon nanotubes [12].

\section{DISCUSSION}

The possibility to develop Fully-Electronics biochips for Label-Free DNA detection has been already demonstrated [28]. In this section, a discussion on the possibility to develop a fully-electronics biochip for multi-panel drugs detection will be carried out taking into account the results of the present study.

A. Peaks Separation by Fitting

The components decomposition of registered peaks in multipanel drugs detection is a hard task, as clearly demonstrated both by simulations (Figure 3) and by experiments (Figure 4). Figure 3 is obtained by considering drugs in close proximity as compounds concentrations: 20, 20, and $15 \mathrm{mM}$ for TM, DC, $\mathrm{SW}$, respectively. However, simulations have clearly shown that different drugs contributions are closely superposed each other even in case of different amount ratios. 


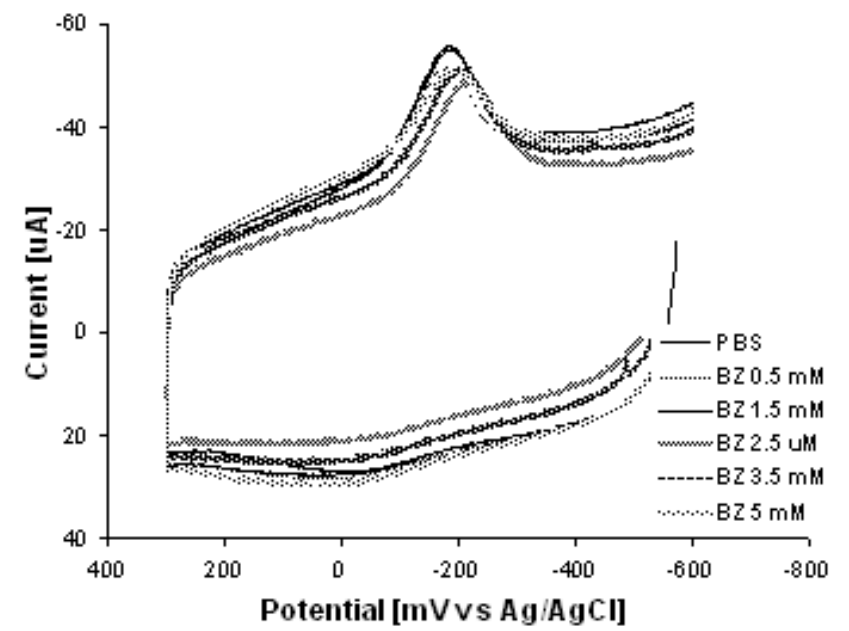

Fig. 5. Voltammogram acquired with P450 2B4 onto Multi Walled Carbon Nanotubes during the detection of Benzphetamine (BZ).

On the other hands, signal-to-noise ratio in real experiments of multiple drugs detection has demonstrated that fittings considering actual positions of each individual drug components are possible (Figure 4). Fitting return us current value for the single drug contribution which does not correspond to calibrations curve as registered in single drug experiments (such as in Figure 6). This is because presence of more than one drug onto the biochip spots means lesser amount of probe proteins for the same drug as the probes surface is partially occupied by the other drugs. Therefore, a correct drugs detection calibration must accounts for a family of curves, each related to different amount of the co-detected drugs.

\section{B. Enzymes Kinetics}

Enzymes kinetics on different target drugs plays a crucial role in designing a multi-panel biochip. In fact, both simulations and experiments shown in this study are related to $\mathrm{P} 450$ substrates kinetics which are close each other. In this case, different contributions to a voltammetric peak are quite equally distributed between the different detected drugs. Therefore, the same spot functionalized with the same P450 have the chance to detect different drugs that are contemporary present in the same sample. However, a drug contribution may be hided by those of other drugs contemporary present in the same sample in case it has highly different kinetics. This is the case, for example, of tolbutamide (TB). This compound is detected by the cytochrome P450 2C9 as well as torsemide (TM), diclofenac (DC), and S-Warfarin (SW). The Km are 11.4 and $6.8 \mathrm{mM}$ for TM and DC [23], and it is equal to $6 \mathrm{mM}$ for SW [24], while it is equal to $120 \mathrm{mM}$ for TB [29]. The $\mathrm{Km}$ is defined as the molar concentration at which the enzymatic reaction reach half of the max velocity. Thus, higher $\mathrm{Km}$ means smaller enzymatic velocity. This is reflected by a smaller current in electrochemical detection. Current registered in cyclic voltammograms is neither trivially nor linearly related to $\mathrm{Km}$. The above reported numbers suggest us that contribution from $\mathrm{TB}$ will be hidden in case of drugs cocktails also involving TM, DC, SW.

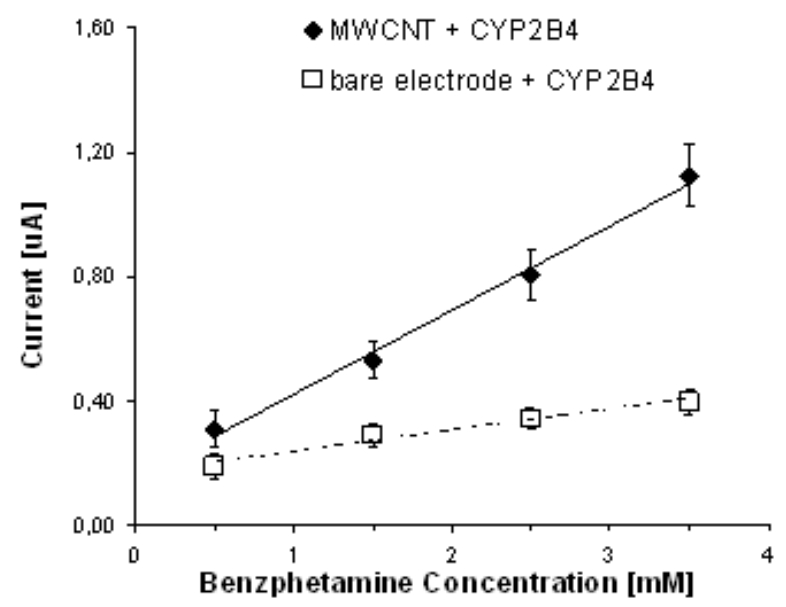

Fig. 6. Currents acquired at fixed potential for different concentration of Benzphetamine (BZ) with or without Multi Walled Carbon Nanotubes

\section{Drug Identification}

Components separation in the voltammetric peaks is, thus, a crucial task of the biochip in case of multi-panel detection. Moreover, it cannot avoid considering extra information about the proposed sample. For example, data set shown in Figure 4 should be explained in terms of both $\mathrm{CP}$ and DX drugs with a chi-square equal to 2.32. However, it also may be explained considering only contribution from a single drug. In that case, the fitting chi-square is higher in case of fitting with only $\mathrm{CP}$ (4.25) but close to the previous one in case of DX (2.27).

Signal-to-noise ratio does not authorize us to consider actually different the two numbers from fitting with $\mathrm{DX}+\mathrm{CP}$ and from only the DX contribution, but the two numbers are really very close each other. However, the two drugs kinetics are so close $[23,24]$ that the right fitting is that considering both DX and $\mathrm{CP}$. Therefore, both the information of the combined presence of the two drugs into the sample and those related the drugs kinetics have to be considered before deciding which drugs are present once acquired by the single cyclic voltammogram.

\section{Software tools for biochip design}

As we have seen, a biochip for point-of-care technology based on the enzymes family $\mathrm{P} 450$ should be a very powerful platform for personalization of drug therapy because of the key role of P450. However, different P450 isoforms may have the same drug compound as substrate and different drugs may be substrates of the same $\mathrm{P} 450$ protein. Proper strategies to develop P450 based biosensor arrays and to query each single array spot, while at the same time maintaining high reliability and low cost of experimentation is highly desired. A possible array design to immobilize P450s for sensing multiple drugs have to consider redundancy and multiple enzyme-substrate interactions. In our approach, the readout is based on selecting the combinations that can distinguish the targets. This selection can be achieved by solving a covering problem[30]. An example is shown in Table 1 where a set of drugs $\{\mathrm{BZ}$; PX; AP; CP; DX; MZ; TM; DC; SW $\}$ is mapped into the cytochromes set $\{2 \mathrm{~B} 4 ; 2 \mathrm{C} 9 ; 3 \mathrm{~A} 4\}$. A bit more complicated example is shown in Figure $7 \mathrm{a}$. In Figure $7 \mathrm{~b}$, the $\mathrm{e}_{5}, \mathrm{e}_{9}, \mathrm{e}_{8}$ and $\mathrm{e}_{10}$ are the minimum number of probes. 


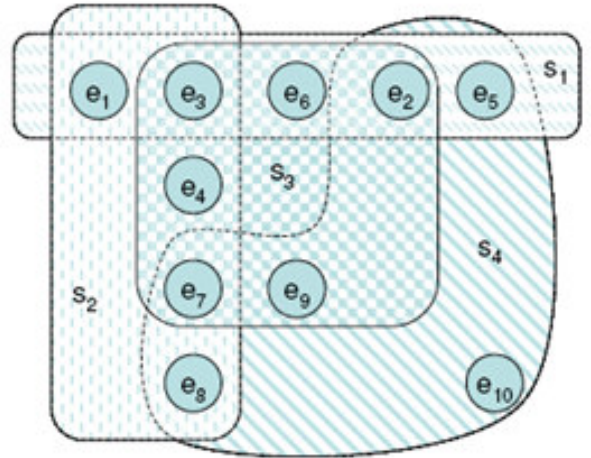

a.

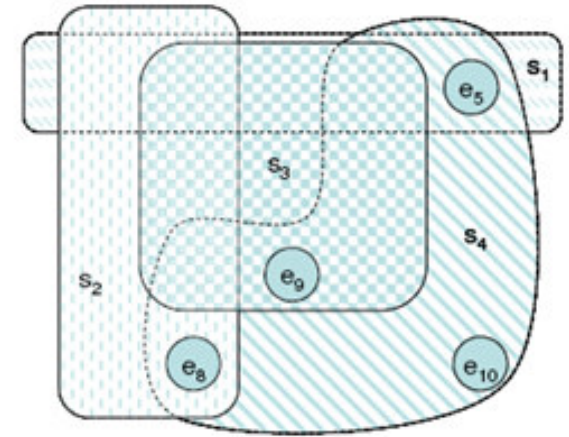

b.

Figure 7: A set cover representation of P450 based biochip design. In Figure a), the bounded boxes correspond to substrates and the disks to interacting enzymes. In Figure b), a subset of enzymes selected by the software that will be used to distinguish all the considered drug components in a mixture.

A simple spot query at fixed potential like those in Figure 6 is proposed for the following pairs $\left\{\mathrm{e}_{5}, \mathrm{~s}_{1}\right\},\left\{\mathrm{e}_{8}, \mathrm{~s}_{2}\right\},\left\{\mathrm{e}_{9}, \mathrm{~s}_{3}\right\}$, $\left\{\mathrm{e}_{10}, \mathrm{~s}_{4}\right\}$. A bit more complicated dynamical spot query like those in Figures 3 and 4 and further signal analysis for peaks separation is proposed to distinguish $\left\{\mathrm{e}_{5}, \mathrm{~s}_{4}, \mathrm{~s}_{1}\right\},\left\{\mathrm{e}_{9}, \mathrm{~s}_{4}, \mathrm{~s}_{3}\right\}$ and $\left\{\mathrm{e}_{8}, \mathrm{~s}_{4}, \mathrm{~s}_{2}\right\}$ by voltammograms signature. If $\mathrm{s}_{4}$ is found to be absent in the mixture (absence of signal on $\mathrm{e}_{10}$ ) then the signal on probes $e_{5}, e_{9}$ and $e_{8}$ is enough to prove the presence of $s_{1}, s_{3}$ and $s_{2}$ respectively without using the signature information, and quantification may be done with calibration curves like one in Figure 4. If $\mathrm{e}_{10}$ probe detects the presence of $s_{4}$ the software dynamically guides the sensor to make a scan for second potential range as defined by voltammogram signature to distinguish between $\left\{\mathrm{s}_{4}, \mathrm{~s}_{1}\right\}$, $\left\{\mathrm{s}_{4}, \mathrm{~s}_{3}\right\}$ and $\left\{\mathrm{s}_{4}, \mathrm{~s}_{2}\right\}$. Algorithms based on irredundant cover computation can be used to add this kind of artificial intelligence to the biosensor chip array.

\section{CONCLUSIONS}

In the present paper we investigated a multi-panel technology that may provide real-time information on multidrugs metabolism. Such a system can enable early identification of drug candidates. Moreover, it may provide innovative solution for biochip for pharmacokinetic studies in animals and humans by shortening the typically 1-2 months now available with current methods (e.g., LCMS/MS). Because some of the studies conducted are sequential, an optimal use of such a multi-panel biochip may accelerate drug development by several months, and it will save costs for analytics by an order of magnitude of $1 \mathrm{M} €$. The potential socio-economical relevance of the biochip systems here proposed is huge for the worldwide society in order to improve the quality of life of the patients, in order to decrease the medical care costs, in order to shorten the time-to-market for drug producers, and in order to increase enormously the diagnostic market. However, this study has clearly outlined the bottleneck and drawbacks that one could face while developing such a biochip. A fully-electronics biochip for multi-panel drugs detection implementing enough intelligence to account for these problems is currently under investigation in our lab in order to verify the possibility to overcome all the drawbacks.

\section{ACKNOWLEDGMENT}

S.C. thanks Uwe Furh for useful discussions on personalized therapies and electrochemistry of P450. Victoria Shumyantseva, from Russian Academy of Biomedical Science, is acknowledged for supplying P450 2B4 and Benzphetamine. The research is financially supported by the EPFL - Integrated Center SI.

\section{REFERENCES}

[1] J. Lazarou, B.H. Pomeranz, Corey P.N, JAMA.279 (1998)1200-5

[2] D. Frank et al., Eur J Clin Pharmacol (2007) 63:321-333

[3] S. T. Turner; et al., Hypertension.50 (2007)1-5;

[4] A.K. Daly Curr Opin Drug Discov Devel.10 (2007) 29-36

[5] http://www.amplichip.us/

[6] Jiunn H. Lin, Current Drug Metabolism 8 (2007) 109-136

[7] S. Joseph, et al., Biochemical Pharmacology,. 65 (2003) 1817-1826.

[8] S. Liu, et al., Analytical Biochemistry 375 (2008) 209-216.

[9] E. Iwuoha, et al., IET Nanobiotechnology, 1 (2007) 62-67

[10] L. Peng, et al., Electroanalysis, 20 (2008) 803-807.

[11] V. Shumyantseva, et al., Biosensors \& Bioelectronics, 21 (2005) 217 222

[12] S. Carrara, et al., Biosensors and Bioelectronics, 24(2008) 148-150.

[13] Murray M \& Petrovic N. Curr Opin Mol Ther. 8 (2006) 480-6

[14] Danielson P Curr Drug Metab 3 (2002) 561-97

[15] Bertz RJ, Granneman GR, Clin Pharmacokinet 32(1997) 210-258

[16] Kirchheiner J, et al. Mol Psychiatry 9 (2004) 442-473

[17] Gasche Y, N Engl J Med 351(2004) 2827-2831

[18] Mahgoub A, et al. Lancet 2(1977) 584-586

[19] Zanger UM, Naunyn-Schmiedeberg's Arch Pharmacol, 369(2004) 23-37

[20] Bertilsson L, Br J Clin Pharmacol 53(2002) 111-122

[21] U. Fuhr et al., Clin Pharmacol Ther 81(2007) 270-83

[22] A.J. Bard, L.R. Faulkner, Electrochemical Methods, $2^{\text {nd }}$ ed., N.Y. 2001, pag 244.

[23] D.L. Johnson, et al., Biochemical Pharmacology, 69, (2005) 15331541

[24] Haining RL.,Biochemistry; 38, (1999) 3285-92.

[25] Song Ren, et al., Cancer Research 7 (1997) 4229-4235

[26] Yi Wang et al., Biopharm. Drug Dispos. 20 (1999) 341-346

[27] V.V. Shumyantseva, et al., J. of Inorg. Biochemistry, 101,2007, 859865

[28] C. Stagni, et al., IEEE Sensor Journal 7 (2007) 577-585

[29] Miners JO, et al., Methods Enzymol 272(1996), 139-45

[30] De Micheli G., Synthesis and Optimization of digital circuits, McGraw-Hill, 1994 